THE

PERFECT

FASCIST 



\section{THE \\ PERFECT FASCIST}

A Story of Love, Power, and Morality in Mussolini’s Italy

\section{VICTORIA DE GRAZIA}

THE B E L KNAP PRES OF

HARVARD U N IVERSITY PRESS

Cambridge, Massachusetts and London, England 2020 


\section{Copyright () 2020 Victoria de Grazia}

\section{ALL RIGHTS RESERVED}

Printed in the United States of America

\section{First printing}

Cover design: Jill Breitbarth

Jacket photos: (top) Bettmann/Getty Images; (bottom) Bettmann/Getty Images

$$
\begin{aligned}
& 9780674245266 \text { (EPUB) } \\
& 9780674245440 \text { (MOBI) } \\
& 9780674245471 \text { (PDF) }
\end{aligned}
$$

The Library of Congress has cataloged the printed edition as follows:

Names: De Grazia, Victoria, author.

Title: The perfect fascist : a story of love, power, and morality in Mussolini’s Italy/Victoria de Grazia.

Description: Cambridge, Massachusetts : The Belknap Press of Harvard University Press, 2020. | Includes bibliographical references and index.

Identifiers: LCCN 2019054630 | ISBN 9780674986398 (hardcover)

Subjects: LCSH: Teruzzi, Attilio, 1882-1950. | Mussolini, Benito, 1883-1945. |

Fascists-Italy-Biography. | Politicians-Italy-Biography. |

Italy-Armed Forces-Officers-Biography. | Italy-History-1922-1945.

Classification: LCC DG575.T38 D4 2020 | DDC 945.091092 [B]-dc23

LC record available at https://lccn.loc.gov/2019054630 
For my beloved brothers, who died on the home front.

For my dearest father, who was always at war. 
